\title{
Identification of Spinning Dust in Ha-Correlated Microwave Emission
}

\section{Citation}

Dobler, Gregory, and Douglas P. Finkbeiner. 2008. "Identification of Spinning Dust in HaCorrelated Microwave Emission." The Astrophysical Journal 680 (2) (June 20): 1235-1242. doi:10.1086/587863.

\section{Published Version}

$10.1086 / 587863$

\section{Permanent link}

http://nrs.harvard.edu/urn-3:HUL.InstRepos:33462896

\section{Terms of Use}

This article was downloaded from Harvard University's DASH repository, and is made available under the terms and conditions applicable to Other Posted Material, as set forth at http:// nrs.harvard.edu/urn-3:HUL.InstRepos:dash.current.terms-of-use\#LAA

\section{Share Your Story}

The Harvard community has made this article openly available.

Please share how this access benefits you. Submit a story.

Accessibility 


\title{
IDENTIFICATION OF SPINNING DUST IN H $\alpha$-CORRELATED MICROWAVE EMISSION
}

\author{
Gregory Dobler $^{1}$ and Douglas P. Finkbeiner ${ }^{1}$ \\ Received 2007 August 3; accepted 2008 February 19
}

\begin{abstract}
CMB experiments commonly use maps of $\mathrm{H} \alpha$ intensity as a spatial template for Galactic free-free emission, assuming a power law $I_{\nu} \propto \nu^{-0.15}$ for the spectrum. Any departure from the assumed free-free spectrum could have a detrimental effect on determination of the primary CMB anisotropy. We show that the $\mathrm{H} \alpha$-correlated emission spectrum in the diffuse WIM is not the expected free-free spectrum at WMAP frequencies. Instead, there is a broad bump in the spectrum at $\sim 50 \mathrm{GHz}$ which is consistent with emission from spinning dust grains. Spectra from both the full sky and smaller regions of interest are well fit by a superposition of a free-free and "warm ionized medium" Draine \& Lazarian spinning dust model, shifted in frequency. The spinning dust emission is $\sim 5$ times weaker than the free-free component at $50 \mathrm{GHz}$, with the null hypothesis that the $\mathrm{H} \alpha$-correlated spectrum is pure free-free ruled out at $\geq 8 \sigma$ in all regions and $>100 \sigma$ for the full-sky fit.
\end{abstract}

Subject headings: diffuse radiation — dust, extinction — ISM: clouds — radiation mechanisms: nonthermal — radio continuum: ISM

\section{INTRODUCTION}

During the course of measuring the cosmic microwave background (CMB) anisotropy, the Wilkinson Microwave Anisotropy Probe (WMAP) has produced the most detailed and sensitive maps of interstellar medium (ISM) emission between 20 and $100 \mathrm{GHz}$ taken to date. The quality of the data has profoundly influenced our understanding of the four main ISM continuum emission mechanisms in this frequency range (Bennett et al. 2003; Finkbeiner 2003; Hinshaw et al. 2007; Dobler \& Finkbeiner 2008). At the highest WMAP frequencies, the Galaxy is dominated by thermal dust emission, traced by far-IR maps (Finkbeiner et al. 1999). At lower frequencies, synchrotron radiation from supernova shockaccelerated electrons spiraling in the Galactic magnetic field makes up the majority of the emission. In the inner Galaxy, the synchrotron emission is brighter relative to low-frequency maps, and has a harder spectrum. ${ }^{2}$ Next, free-free emission from unbound electrons interacting with ions in $\sim 10^{4} \mathrm{~K}$ ionized gas has a harder spectrum than synchrotron, and is detectable in all bands. Finally, there is a bump in the spectrum of dust-correlated emission at $\sim 20 \mathrm{GHz}$, sometimes called "Foreground X" (de Oliveira-Costa et al. 2002). Over the last decade, it has been recognized that this could be electric dipole emission from rapidly rotating dust grains ("spinning dust"; Draine \& Lazarian 1998).

\subsection{Foreground X: Spinning Dust?}

Spinning dust is the electric dipole emission from the smallest dust grains which have a nonzero electric dipole moment and are spun up by several different interactions with the interstellar medium (e.g., ion collisions, the interstellar radiation field, etc.; see Draine \& Lazarian 1998). Spinning dust was considered as a potential source of radio and microwave emission in other contexts (Erickson 1957; Ferrara \& Dettmar 1994). Because the model

\footnotetext{
1 Institute for Theory and Computation, Harvard-Smithsonian Center for Astrophysics, 60 Garden Street, MS-51, Cambridge, MA 02138; gdobler@cfa .harvard.edu,dfinkbeiner@cfa.harvard.edu.

2 Finkbeiner (2004) has previously interpreted this as a separate component, the synchrotron "haze," but this interpretation is controversial (Finkbeiner 2004; Dobler \& Finkbeiner 2008). This haze component is spatially orthogonal to the other templates by construction, and although we include it in our fits below, its existence has negligible effect on the $\mathrm{H} \alpha$-correlated spectrum.
}

depends on the grain size distribution, ionization fraction, electric dipole moments, etc., the peak frequency can be anywhere from $10-50 \mathrm{GHz}$, but is usually in the $20-40 \mathrm{GHz}$ range. This dependence of the spectrum on the ISM parameters has made spinning dust difficult to definitively identify, but may provide the qualitative behaviors necessary to make a foolproof detection.

In the COBE DMR data, Kogut et al. (1996) found that the dust-correlated emission does not simply fall off monotonically with decreasing frequency as would be expected for a thermal dust tail, but instead was greater at $31 \mathrm{GHz}$ than at $53 \mathrm{GHz}$. This behavior was confirmed by the Saskatoon CMB experiment (de Oliveira-Costa et al. 1997), the 14.5 and $32 \mathrm{GHz}$ OVRO data (Leitch et al. 1997), the Cottingham and Boughn $19.2 \mathrm{GHz}$ survey (de Oliveira-Costa et al. 1998), the Tenerife 10 and $15 \mathrm{GHz}$ survey (de Oliveira-Costa et al. 1999), and the QMAP $30 \mathrm{GHz}$ data (de Oliveira-Costa et al. 2000). A small survey of dust clouds with the Green Bank $42 \mathrm{~m}$ telescope at 5, 8, and $10 \mathrm{GHz}$ (Finkbeiner et al. 2002) revealed two candidates for spinning dust clouds: L1622, later confirmed (Finkbeiner 2004); and LPH 201.663+1.643, which turned out to be incorrect (Dickinson et al. 2006). Given this evidence, it was already clear prior to the WMAP data release in early 2003, that there was some nonstandard emission mechanism, but it was unclear whether this mechanism was spinning dust (Draine \& Lazarian 1998) or some other process.

WMAP (Bennett et al. 2003) found that the dust-correlated emission falls from 94 to $61 \mathrm{GHz}$ and then rises steadily to $23 \mathrm{GHz}$. The fact that synchrotron typically dominates at lower frequencies led Bennett et al. (2003) to interpret the low frequency rise as "dust-correlated synchrotron." The reasoning was that because dusty regions correlate with regions of star formation activity, electrons in the vicinity could be accelerated by supernova shocks leading to synchrotron emission. Finkbeiner (2004) pointed out that WMAP is also consistent with Draine \& Lazarian (1998), if their model could be shifted down in frequency a bit.

Because the WMAP frequency coverage (23-94 GHz) coincides with the range where the spinning dust and synchrotron spectra are similar, data at lower frequencies, where the spectra diverge, have been used to test the dust-correlated synchrotron hypothesis. The $W M A P$ "synchrotron" template has a much weaker crosscorrelation at $10-15 \mathrm{GHz}$ than expected for actual synchrotron (de Oliveira-Costa et al. 2004). Also, 8 and $14 \mathrm{GHz}$ data from 
the Green Bank Galactic Plane Survey (Langston et al. 2000) show a rising spectrum consistent with spinning dust but not synchrotron (Finkbeiner et al. 2004) and imply that the majority of dust-correlated emission at $23 \mathrm{GHz}$ is Foreground X. Comparing the Cottingham and Boughn survey to WMAP also shows a rise from 19 to $23 \mathrm{GHz}$ (Boughn \& Pober 2007). The polarization properties (Page et al. 2007; Hinshaw et al. 2007) in the threeyear $W M A P$ data also favor the spinning dust hypothesis.

In spite of all this evidence, there are weaknesses in each of these arguments. Some use only low-latitude data and may not apply to the diffuse ISM (Finkbeiner et al. 2004). Others apply to only one cloud (Finkbeiner et al. 2002), have a poor signal-tonoise ratio (de Oliveira-Costa et al. 2004), or a short lever arm in frequency (Boughn \& Pober 2007). However, taken together, they are very strong evidence against the dust-correlated synchrotron hypothesis, but they are not specifically evidence for spinning dust. Other mechanisms such as magnetic dipole dust emission, caused by the thermal fluctuation of the magnetization in the dust grains (Draine \& Lazarian 1999), could conceivably explain all these results. This ambiguity motivated our search for further clues in the WMAP three-year data.

\subsection{WMAP Multilinear Regression}

In Dobler \& Finkbeiner (2008, hereafter DF08), we describe our method for determining the spectral shape of each of the four primary $W M A P$ foreground components: free-free, dust, soft synchrotron, and hard synchrotron (termed the "haze" by Finkbeiner 2004) emission. We model the WMAP data as a linear combination of the CMB and the four foregrounds, each described by a specific spatial template. For free-free emission we use the $\mathrm{H} \alpha$ map described in $\S 2.1$, for dust (thermal and spinning) emission we use the Schlegel et al. (1998) dust map evaluated at $94 \mathrm{GHz}$ by Finkbeiner et al. (1999), for soft synchrotron we use the Haslam et al. (1982) $408 \mathrm{MHz}$ map, and for the haze we use a simple $1 / r$ template where $r$ is the distance from the Galactic center. The individual $W M A P$ bands were completely decoupled in our fit, and we assumed nothing about the spectrum of each foreground, only that its morphology was traced by the template in all bands. We performed our fits over both the (nearly) full sky and also on smaller regions of interest. Point sources and regions where dust extinction makes the $\mathrm{H} \alpha$ map unreliable [where $A(\mathrm{H} \alpha)=$ $2.65 E(B-V)>1 \mathrm{mag}]$ were masked out from the fits.

We showed that the spectrum of $\mathrm{H} \alpha$-correlated emission is not the usual free-free spectrum. Rather, there is a bump around $\nu \sim$ $50 \mathrm{GHz}$. In $\S 2.3$ we explain why the $\mathrm{H} \alpha$ map should actually trace spinning dust excited by collisions with ions. In $\S 3$ we present $\mathrm{H} \alpha$-correlated emission spectra for both the (nearly) full sky as well as smaller regions of interest and show that, indeed, the spectra are well characterized as a superposition of free-free and spinning dust type spectra. We summarize this work and discuss implications for future observations in $\S 4$.

\section{EMISSION MEASURE AS A TRACER OF SPINNING DUST}

In this section we argue that in fully ionized environments, the emissivity density $\left(j_{\nu}\right)$ of spinning dust emission is proportional to dust density times some excitation function related to the ion density. The emission comes from the very smallest grains, which are spun up by a collision with an ion and spin down as they radiate. In the limit where the spin-down time is small compared to the time between collisions, the rotation is episodic and the emissivity also scales linearly with the ion number density. In $\S 2.2$, we assume the episodic limit in order to build some intuition about how WIM-correlated spinning dust might behave.

\subsection{Free-Free Emission Measure}

The map assembled by Finkbeiner (2003) from the VTSS (Dennison et al. 1998), SHASSA (Gaustad et al. 2001), and WHAM (Haffner et al. 2003) surveys traces the $\mathrm{H} \alpha$ recombination line emission from hot interstellar gas. At $W M A P$ frequencies, this $\mathrm{H} \alpha$ map $^{3}$ is used to estimate the thermal bremsstrahlung, or free-free emission, resulting from the interaction of the electrons and protons from ionized hydrogen (Bennett et al. 2003; Finkbeiner 2004; Hinshaw et al. 2007). The $\mathrm{H} \alpha$ map is corrected for dust absorption using the prescription given by Finkbeiner (2003; but see Dickinson et al. 2003, for an alternative). In this frequency range the free-free specific intensity $I_{\nu}$ (in $\mathrm{Jy} \mathrm{sr}^{-1}$ ) is often approximated as a power law,

$$
I_{\nu} \propto \nu^{\alpha}
$$

with $\alpha \approx-0.15$. Although this is a good approximation, in our analysis we use the expression given by Spitzer (1978) for the free-free spectrum.

Recombination line emission is a two-particle process (involving the capture of free electrons by a proton nucleus) and so the emissivity is proportional to $n_{e} n_{i}$, which for fully ionized $\mathrm{H}$ is simply the square of the number density of gas particles, $j_{\mathrm{H} \alpha} \propto n^{2}$. Therefore, the $\mathrm{H} \alpha$ intensity corresponds to an emission measure (EM) defined as $E_{m} \equiv \int n^{2} d l$, where the integral is taken along the line of sight. The $\mathrm{H} \alpha$ intensity is usually measured in Rayleighs $\left(1 \mathrm{R} \equiv 10^{6} / 4 \pi\right.$ photons s $\left.\mathrm{cm}^{-1} \mathrm{sr}^{-1}\right)$ with an $\mathrm{EM}$ of $1 \mathrm{~cm}^{-6} \mathrm{pc}$ $\approx 0.6 \mathrm{R}$ for gas at $\sim 8000 \mathrm{~K}$.

\subsection{Spinning Dust Emission Measure}

Draine \& Lazarian (1998, hereafter DL98) present a model for spinning dust emission in which tiny dust grains with nonnegligible electric dipole moment are spun up by ion collisions; polycyclic aromatic hydrocarbons (PAHs) generally have a nonzero dipole, because perfect symmetry is rare in large molecules. DL98 estimated the astronomical value of this parameter (actually the electric dipole moment per root mass) from measured electric dipole moments for dozens of known PAH molecules. In the limit where ion interactions provide the dominant spin-up mechanism (see DL98 for details) and assuming full ionization, constant gas/dust ratio, and the same grain size distribution everywhere, the resulting total spinning dust emission is $I \equiv \int I_{\nu} d \nu \propto$ $\int n_{i} n_{d} d l \propto \int n_{i}^{2}$. With the additional assumption that the emission is episodic, the spectrum of the emission does not vary with $n_{i}$, but the amplitude does. That is, the spinning dust emission should look like an EM, and be traced by $\mathrm{H} \alpha$. This approximation is relevant where the collisional excitation is primarily due to ions (at least for the smallest grains), e.g., for the "warm ionized medium" (WIM) parameters used by DL98.

The WIM-correlated emission claimed here augments the "usual" spinning dust emission in the "cold neutral medium" (CNM) which has a temperature $T_{\mathrm{CNM}} \sim 100 \mathrm{~K}$ and is traced by the Schlegel et al. (1998) dust map evaluated at $94 \mathrm{GHz}$ by Finkbeiner et al. (1999, FDS99 in Fig. 5, below).

\subsection{Estimating the Specific Intensity}

For a spherical dust grain of size $a$, rotating with angular frequency $\omega$, and with a dipole moment $p_{0}$, the rotational energy is

$$
E=\frac{1}{2} I \omega^{2}=\frac{1}{5} m a^{2} \omega^{2},
$$

\footnotetext{
3 Available at http://www.skymaps.info.
} 

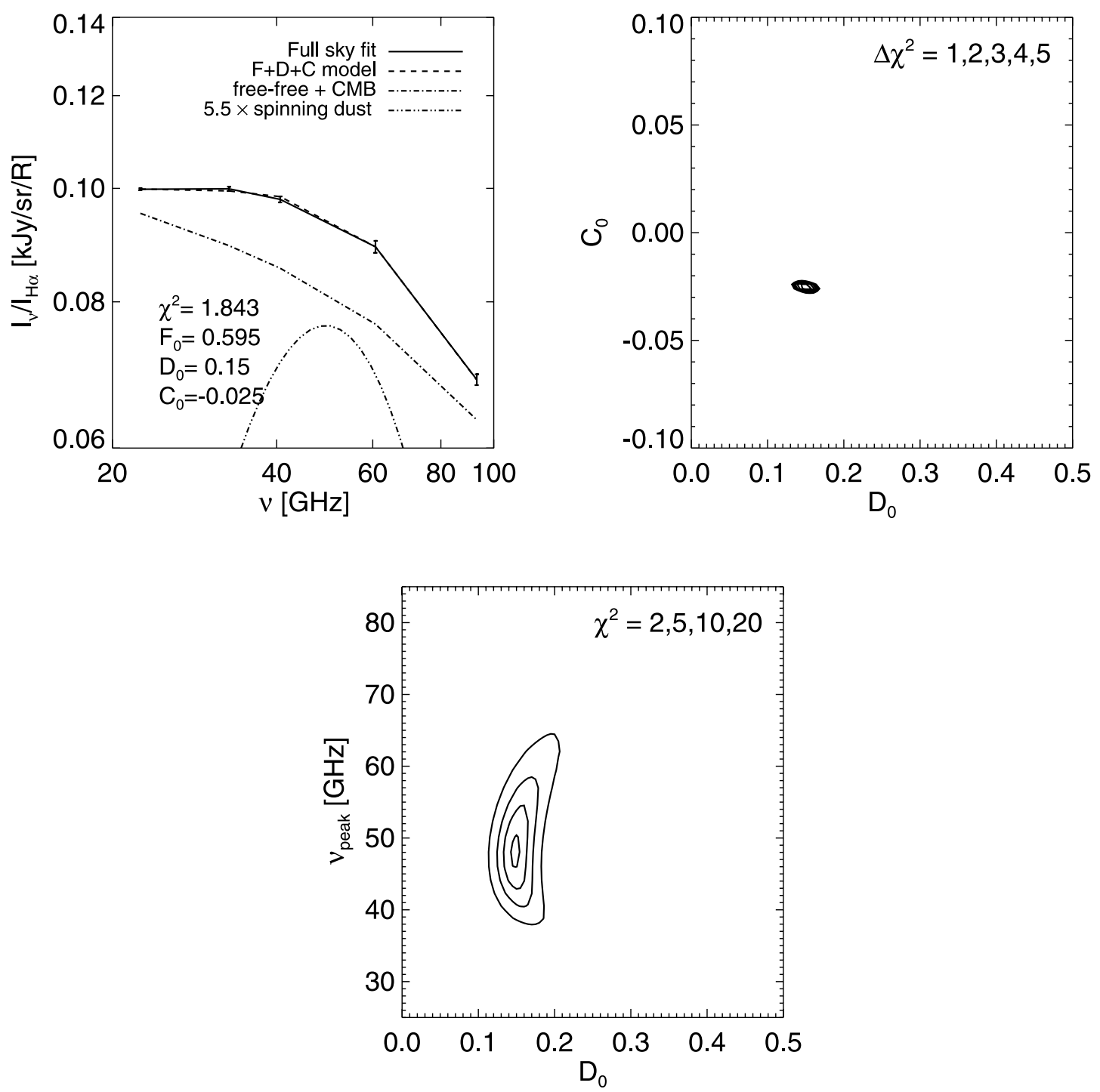

FIg. 1.-Top left: Best $F+D+C$ (free-free plus spinning dust plus CMB) model fit to the H $\alpha$-correlated emission over the full sky. The peak frequency is fixed to $\nu_{\text {peak }}=50 \mathrm{GHz}$. This two-component plus CMB contamination model works surprisingly well with a spinning dust component that is 6.23 times weaker than the free-free (see Table 1). Top right: $\Delta \chi^{2}$ contours for the best-fit free-free coefficient in the spinning dust/CMB plane. The null hypothesis that the H $\alpha$-correlated emission can be well fit by only a $F+C$ model (i.e., $\left.D_{0}=0\right)$ is ruled out at very high confidence $(335 \sigma)$. Bottom: $\chi^{2}$ contours in the spinning dust- $\nu_{\text {peak }}$ plane. Although the peak frequency is not very well constrained, we can rule out $\nu_{\text {peak }}<40 \mathrm{GHz}$ and $\nu_{\text {peak }}>60 \mathrm{GHz}$ at $>4 \sigma$ confidence.

where $m$ is the grain mass. The energy loss rate is the total power emitted by the grain,

$$
\dot{E}=\frac{1}{12 \pi} \frac{\mu_{0}}{c} p_{0}^{2} \omega^{4}
$$

and the spin-down time is

$$
\tau_{s} \equiv E / \dot{E} \propto a^{2} p_{0}^{-2} \omega^{-2} .
$$

The time between collisions is just

$$
\tau_{c} \sim \lambda / v \propto a^{-2} n_{\mathrm{H}}^{-1},
$$

where $v$ is the thermal velocity of the ion and $\lambda=\left(\pi a^{2} n_{\mathrm{H}}\right)^{-1}$ is the mean free path.

In the limit where the spin-down time is much smaller than the time between collisions $\left(r \equiv \tau_{s} / \tau_{c} \ll 1\right)$, the excitation is episodic.
For the WIM parameters in DL98, this ratio is in fact $\sim 1$; however, we point out that

$$
r=\tau_{s} / \tau_{c} \propto a^{4} n_{\mathrm{H}} p_{0}^{-2} \omega^{-2},
$$

so that very small changes to the grain size distribution can substantially affect the spin-down time compared to the time between collisions. In fact, if each ion collision imparts equal energy, then from equation (2), $\omega \propto a^{-2}$. Since $p_{0} \propto N^{1 / 2} \propto a^{3 / 2}$ (see DL98) then in this case, $1 / r$ diverges as $a^{-5}$. The bottom line is that the episodic approximation is sensitively dependent on the ion density, the dipole moment, and especially the grain size distribution. For the purposes of estimating a specific intensity, we will now assume the episodic approximation with the caveat that it may break down in dense environments (e.g., H II regions; in fact, Scaife et al. [2008] find no evidence for spinning dust at 14.2 and $17.9 \mathrm{GHz}$ in 16 Galactic $\mathrm{H}$ II regions) or for very small dipole moments. We emphasize that this does not affect the fact that the total intensity I should be proportional to $\int n_{d} n_{\mathrm{H}} d \ell$ so that spinning dust emission should be traced by an EM map. 

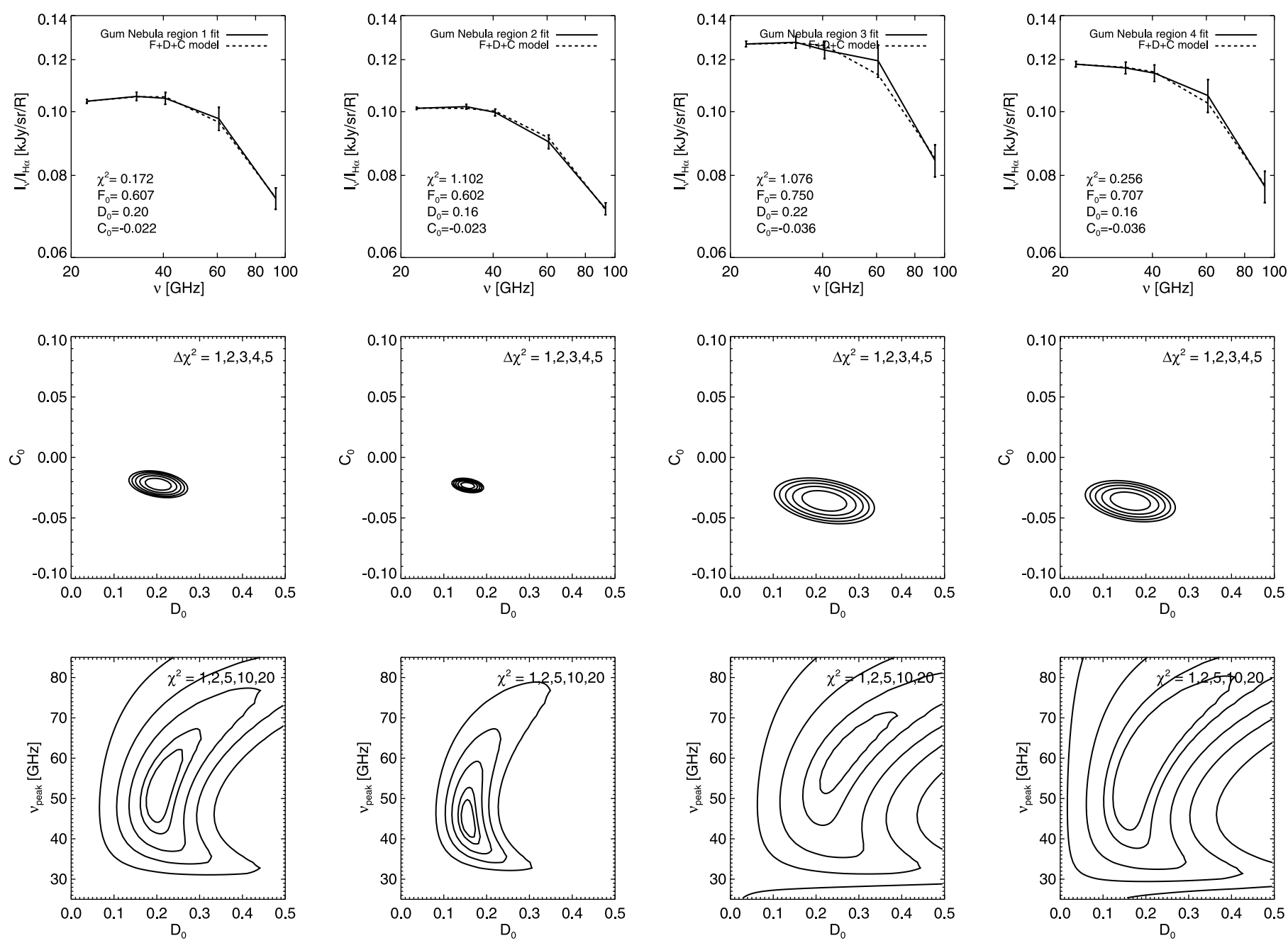

Fig. 2. - Same as Fig. 1, except for the four regions of the Gum Nebula (see Table 1). Although the peak frequency is only loosely constrained, the $D_{0}=0$ null hypothesis is ruled out at $\geq 12 \sigma$ in all regions.

DL98 evaluate $j_{\nu} / n_{\mathrm{H}}$ for parameters representative of various interstellar environments. In the limit that $j_{\nu} / n_{\mathrm{H}}$ is a constant with $n_{\mathrm{H}}$, then it equals $I_{\nu} / N(H)$. However, in our case it is not. As a reference model, we take the DL98 WIM model evaluated for $n_{\mathrm{H}}=0.1 \mathrm{~cm}^{-3}$ which peaks near $25 \mathrm{GHz}$ with a peak emissivity ${ }^{4}$ of $j_{p \text {, ref }} / n_{\mathrm{H}}=5 \times 10^{-21} \mathrm{kJy} \mathrm{sr}^{-1} \mathrm{~cm}^{2}$ or $j_{p \text {,ref }}=5 \times 10^{-22} \mathrm{kJy} \mathrm{sr}^{-1}$ $\mathrm{cm}^{-1}$. Multiplying the dependence on dust and gas density we have ${ }^{5}$

$$
j_{p}=j_{p, \text { ref }}\left(\frac{n_{\mathrm{H}}}{n_{\mathrm{H}, \text { ref }}}\right)\left(\frac{n_{d}}{n_{d, \text { ref }}}\right),
$$

where $n_{\mathrm{H}, \text { ref }}$ and $n_{d \text {, ref }}$ are the density of $\mathrm{H}$ and small dust grains, respectively, used in the reference model. For full ionization of $\mathrm{H}\left(n_{\mathrm{H}}=n_{i}\right)$ and constant gas/dust ratio $\left(n_{\mathrm{H}} / n_{\mathrm{H}, \text { ref }}=n_{d} / n_{d, \text { ref }}\right)$ we have

$$
\begin{aligned}
j_{p} & =j_{p, \text { ref }}\left(\frac{n_{\mathrm{H}}}{0.1 \mathrm{~cm}^{-3}}\right)^{2} \\
& =5 \times 10^{-20} n_{\mathrm{H}}^{2} \mathrm{kJy} \mathrm{sr}^{-1} \mathrm{~cm}^{5} \\
& =0.15 n_{\mathrm{H}}^{2} \mathrm{kJy} \mathrm{sr}^{-1} \mathrm{~cm}^{6} \mathrm{pc}^{-1}
\end{aligned}
$$

\footnotetext{
4 The units of the ordinate, $j_{\nu} / n_{\mathrm{H}}$, of Figs. $9-11$ in DL98 are incorrectly labeled $\mathrm{Jy} \mathrm{sr}^{-1}$ rather than $\mathrm{Jy}_{\mathrm{cm}}^{2} \mathrm{sr}^{-1}$, an error propagated by Finkbeiner et al. (2002).

5 Again, the dependence on $n_{\mathrm{H}}$ in eq. (7) is only strictly correct in the episodic limit, although in reality the deviation is likely small.
}

and the peak specific intensity is then

$$
I_{p}=\int j_{p} d l=0.15 \mathrm{kJy} \mathrm{sr}^{-1}\left(\frac{E_{m}}{1 \mathrm{~cm}^{-6} \mathrm{pc}}\right),
$$

or

$$
I_{p} / I_{\mathrm{H} \alpha}=0.09 \mathrm{kJy} \mathrm{sr}^{-1} \mathrm{R}^{-1},
$$

where $I_{\mathrm{H} \alpha}$ is measured in R. For comparison, the ratio of freefree to $\mathrm{H} \alpha$ for a temperature of $T_{e} \sim 8000 \mathrm{~K}$ at $41 \mathrm{GHz}$ (WMAP Q-band) is $I_{41} / I_{\mathrm{H} \alpha} \sim 0.15 \mathrm{kJy} \mathrm{sr}^{-1} \mathrm{R}^{-1}$.

Although this is a rough estimate, it suggests that the intensity of the free-free emission at $41 \mathrm{GHz}$ and the peak of the spinning dust emission should be of the same order of magnitude. Throughout this discussion we make the assumption that the DL98 WIM spectrum can be shifted by a factor of 2 in frequency. A more thorough analysis would require that the DL98 model be evaluated for some set of parameters (ion density, electric dipole moment per root mass, etc.) that give the actual spectrum observed. For our purposes this naive shift is adequate.

\section{SPECTRUM OF H $\alpha$-CORRELATED EMISSION}

As noted above, in DF08 we showed that the spectrum of $\mathrm{H} \alpha$-correlated emission in the WMAP data has a bump around 

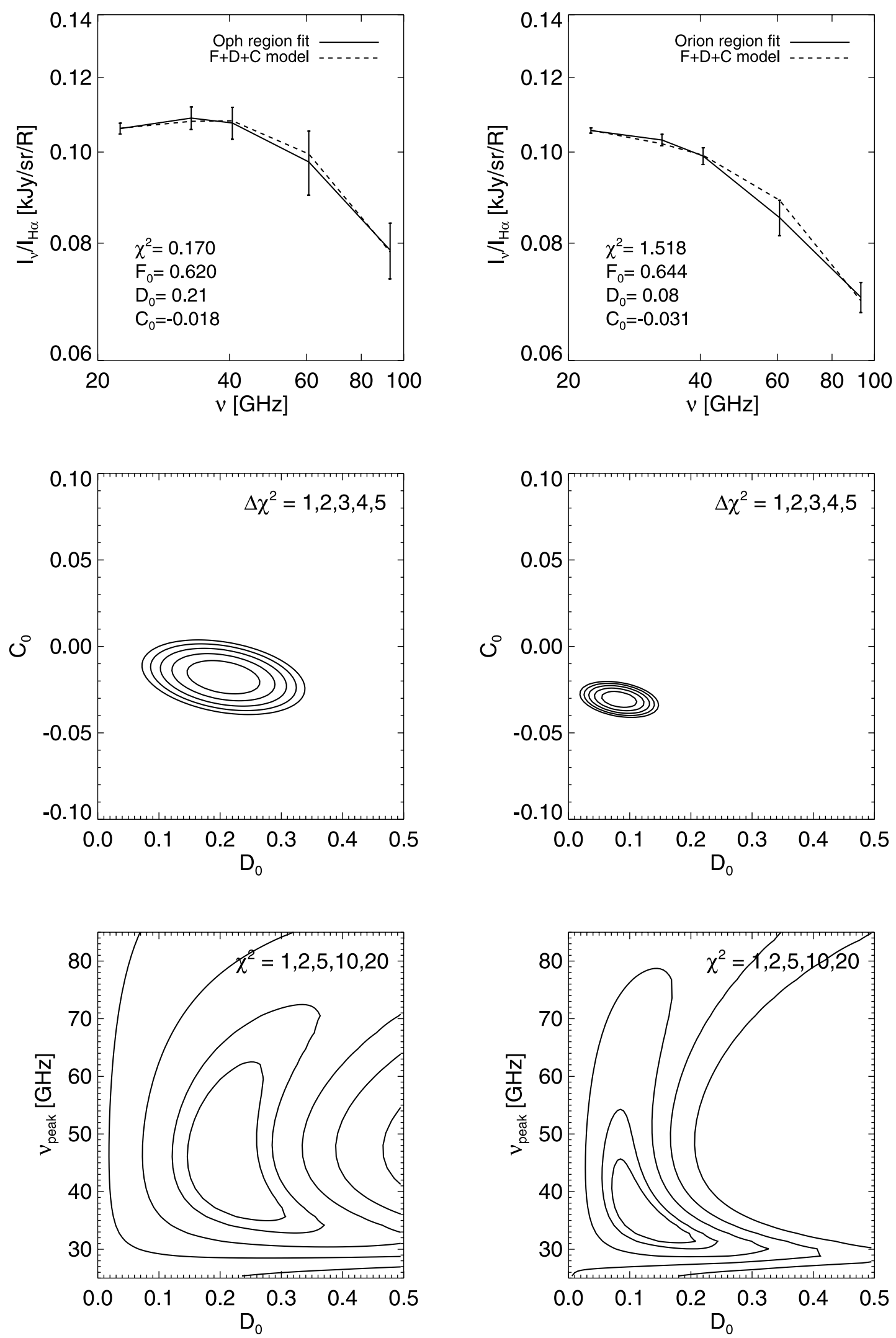

FIG. 3.- Same as Fig. 1, except for a region which encloses the Ophiuchus complex (left) and one which is near the Orion Nebula (right). See Table 1 for details. 
TABLE 1

Region Definitions and Fit Results

\begin{tabular}{|c|c|c|c|c|c|c|c|}
\hline REGION & Full SKY & \multicolumn{4}{|c|}{ Gum Nebula } & Ophiuchus & ORION \\
\hline$l$ range $(\mathrm{deg}) \ldots \ldots \ldots$ & {$[0,360]$} & {$[235,256]$} & {$[256,280]$} & {$[235,256]$} & {$[256,280]$} & {$[334,13]$} & {$[195,230]$} \\
\hline$b$ range $(\operatorname{deg})$ & {$[-90,90]$} & {$[-30,0]$} & {$[-30,0]$} & {$[0,30]$} & {$[0,30]$} & {$[0,90]$} & {$[-35,0]$} \\
\hline$\chi^{2}$ & 1.84 & 0.17 & 1.10 & 1.08 & 0.26 & 0.17 & 1.52 \\
\hline$\Delta \chi_{D=0}^{2}$ & 335.02 & 43.84 & 87.32 & 17.59 & 12.09 & 11.89 & 8.30 \\
\hline$I_{F} / I_{D}\left(\nu=\nu_{\text {peak }}\right)$ & 6.23 & 4.70 & 6.07 & 5.38 & 6.80 & 4.75 & 12.22 \\
\hline
\end{tabular}

Notes.-Region definitions (in Galactic coordinates $l$ and $b$ ) and results of the fits presented in Figs. $1-3$. The very low values of $\chi^{2}$ reflect both the quality of the fits and the small number of constraints (number of degrees of freedom $=2$ ). The $D_{0}=0$ null hypothesis is ruled out at $>8 \sigma$ in all regions, while the relative intensity of free-free to spinning dust emission at $\nu_{\text {peak }}=50 \mathrm{GHz}$ is in the range 4.7-12.2.

$\nu \sim 50 \mathrm{GHz}$ that we now argue is consistent with a spinning dust contamination of the familiar equation (1) free-free spectrum. We also point out in DF08 that any measurement of a foreground spectrum is necessarily contaminated by a component which has the frequency dependence of the CMB due to imperfect foreground removal when constructing a CMB template. ${ }^{6}$ However, as we will show shortly, this contamination is incapable of producing the observed bump.

Figure 1 shows our full-sky fit for $\mathrm{H} \alpha$-correlated emission. Our hypothesis is that this spectrum can be well fit by a linear combination of free-free, spinning dust, and CMB spectra. Thus, our model spectrum is

$$
I_{\nu}^{\bmod }=F+D+C
$$

where

$$
\begin{aligned}
& F=F_{0}\left(\frac{\nu}{23 \mathrm{GHz}}\right)^{-0.15} \\
& D=D_{0}\left(\text { DL98 WIM model with } \nu_{p}=50 \mathrm{GHz}\right) \\
& C=C_{0}\left(\frac{\nu}{23 \mathrm{GHz}}\right)^{2}
\end{aligned}
$$

We minimize $\chi^{2}=\sum_{i}\left(I_{\nu_{i}}^{\bmod }-I_{\nu_{i}}\right)^{2} / \sigma_{i}^{2}$ over the parameters $F_{0}$, $D_{0}$, and $C_{0}$, which are scaled to be of order unity. Here $\sigma_{i}$ are the formal errors in band $i$ of the spectrum from our DF08 template fit, and we have one degree of freedom.

As shown in Figure 1, the spectral model in equation (13) fits the data astonishingly well. The free-free and spinning dust amplitudes are comparable while the contamination from the $\mathrm{CMB}$ is minimal. The very low value of $\chi^{2}=1.84$ reflects the quality of the fit. Figure 1 also shows $\Delta \chi^{2}=1,2,3,4$, and 5 contours in the $D_{0}-C_{0}$ plane minimizing $\chi^{2}$ over $F_{0}$ at each point, and holding $\nu_{\mathrm{p}}$ fixed at $50 \mathrm{GHz}$. The null hypothesis that only a free-free plus $\mathrm{CMB}$ spectrum (i.e., $D_{0}=0$ ) fit the data is ruled out to a very high significance. Thus, either free-free emission does not follow equation (1), or the $\mathrm{H} \alpha$ map is tracing an additional emission component. This other emission component seems to be well fit by a shifted WIM spinning dust spectrum.

Lastly, the $\chi^{2}$ contours in the $D_{0}-\nu_{\text {peak }}$ plane in the bottom panel of Figure 1 indicate that, although the peak frequency of the spinning dust is not very well constrained, values of $\sim 45-55 \mathrm{GHz}$

\footnotetext{
${ }^{6}$ Throughout this paper, we will work with the results found using our CMB5 estimator for the CMB - see DF08 for details.
}

are consistent with the data. Peak frequencies less than $40 \mathrm{GHz}$ and greater than $60 \mathrm{GHz}$ are ruled out at $4 \sigma$.

The next step is to consider smaller individual regions of the sky to show that the signal persists. Figures 2 and 3 show fits for four different regions of the Gum Nebula, the north central Galactic sky (including the Ophiuchus complex), and a region near the Orion Nebula (see Table 1 and Fig. 4 for details). Although the fits are significantly noisier as a result of the smaller number of pixels used, the bump in the $\mathrm{H} \alpha$-correlated emission remains in all regions, with the free-free amplitude roughly 4-7 times larger than the spinning dust. Furthermore, the case of no spinning dust $D_{0}=0$ is ruled out in all regions at $\geq 8 \sigma$. Although the $\chi^{2}$ contours in the $D_{0}-\nu_{\text {peak }}$ plane offer a tantalizing hint that the peak frequency of the spinning dust is lower in the Orion Nebula region than the others, the present data are simply too noisy to make a statistically significant statement.

Lastly, we address the possibility that the bump in the $\mathrm{H} \alpha$ correlated emission represents a cross-correlation bias between the different templates used in DF08. That is to say, perhaps the
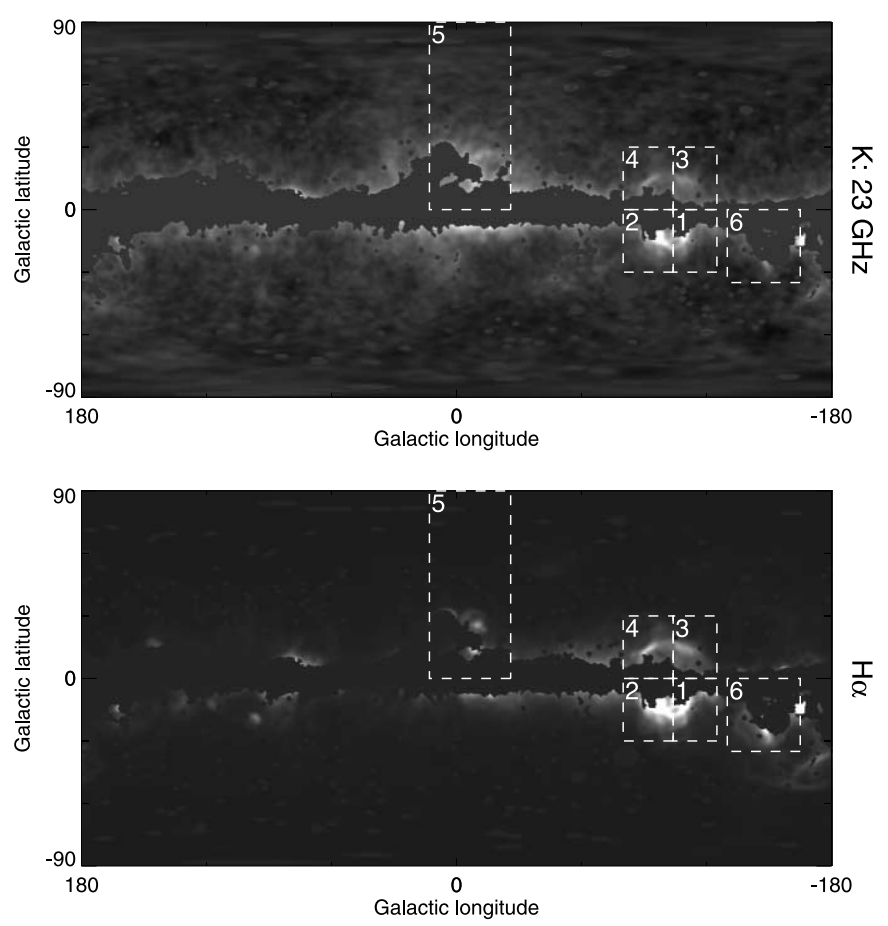

FIG. 4.-WMAP K-band data (top) and the $\mathrm{H} \alpha$ map (bottom) with our different fit regions shown as dotted boxes. In addition to the full-sky fit, the regions are the Gum Nebula (1, 2, 3, and 4), the Ophiuchus complex (5), and the Orion Nebula (6). See Table 1 for details. 
TABLE 2

Correlation Matrix

\begin{tabular}{lcccc}
\hline \hline & $\mathrm{H} \alpha$ & FDS99 & Haslam & Haze \\
\hline H $\alpha$ & 1.00 & 0.45 & 0.18 & 0.10 \\
FDS99 & 0.45 & 1.00 & 0.56 & 0.26 \\
Haslam & 0.18 & 0.56 & 1.00 & 0.58 \\
Haze & 0.10 & 0.26 & 0.58 & 1.00 \\
\hline
\end{tabular}

NoTEs. - The correlation matrix for the templates presented in Dobler \& Finkbeiner (2008). These are the templates that were used in the multilinear regression fit which revealed the bump in the $\mathrm{H} \alpha$-correlated emission presented in Figs. 1-3.

$\mathrm{H} \alpha$ spectrum is absorbing power from other templates in the fit. Table 2 shows the correlation matrix, defined as

$$
\Gamma_{i, j}=\frac{\left\langle T_{i} T_{j}\right\rangle}{\sqrt{\left\langle T_{i}^{2}\right\rangle\left\langle T_{j}^{2}\right\rangle}},
$$

where $\Gamma_{i, j}$ is the correlation between the $i$ th and $j$ th template. The correlation between the templates is rather small. In partic- ular, the dust map is the template which is most highly correlated with the $\mathrm{H} \alpha$ map, and even then, the variance in the $\mathrm{H} \alpha$-correlated emission can be at most $\Gamma_{\mathrm{H} \alpha, \mathrm{FDS} 99}^{2}=20 \%$ due to correlation with dust.

In fact, Figure 5 shows that the effects are much smaller. Here, the solid lines show the resultant spectra for all foreground components both with and without fitting the $\mathrm{H} \alpha$ spectrum. In the latter case the $\mathrm{H} \alpha$ emission was assumed to follow the free-free spectrum in equation (1). The features of these spectra are discussed at length in DF08, but the important point is that the other foregrounds are not significantly affected when the $\mathrm{H} \alpha$ spectrum is fixed. This is strong evidence that the emission mechanism causing the bump in the $\mathrm{H} \alpha$ spectrum is highly correlated with the $\mathrm{H} \alpha$ template.

\section{DISCUSSION}

In Dobler \& Finkbeiner (2008) we identified a deviation from the classical free-free (thermal bremsstrahlung) spectrum in the $\mathrm{H} \alpha$-correlated emission of the three-year WMAP data. In this paper we have argued that the spectral data are consistent with a superposition of free-free and spinning dust emission from a "warm
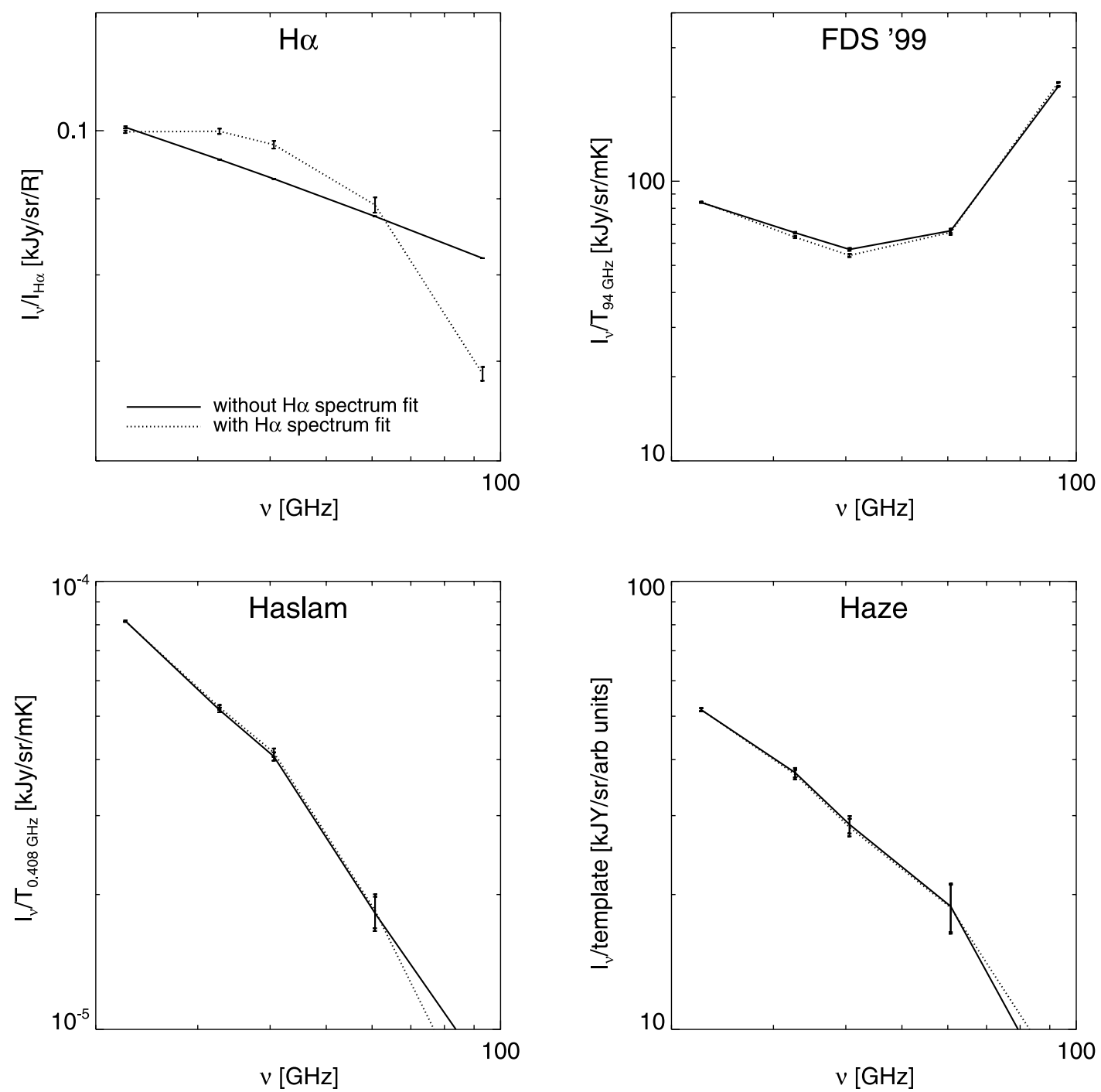

FIG. 5.- Intensity of the emission correlated with the four templates presented in Dobler \& Finkbeiner (2008). The solid lines indicate fits for which the H $\alpha$ correlated emission was constrained to follow a $I_{\mathrm{ff}} \propto \nu^{-0.15}$ (see eq. [1]) frequency dependence, while the dotted lines indicate that the H $\alpha$-correlated spectrum was fit explicitly. Both the insensitivity of the inferred dust-, Haslam-, and haze-correlated spectra to fixing the H $\alpha$ spectrum, as well as the relatively low correlation among the templates ( Table 2), indicates that the spinning dust bump is indeed correlated primarily with $\mathrm{H} \alpha$ emission. 
ionized medium" (WIM; see Draine \& Lazarian 1998). In addition, there is contamination by a component with the spectrum of the $\mathrm{CMB}$ due to chance morphological correlation between the CMB and the $\mathrm{H} \alpha$ map (see Dobler \& Finkbeiner 2008 for details).

Since the intensity of both free-free and WIM spinning dust emission scale proportionately with the number density squared (free-free emission is generated by the collision of free electrons with ions while the WIM spinning dust emission comes from collision of ions with tiny dust grains), both should be traced by an emission measure map like the $\mathrm{H} \alpha$ map. We have assumed throughout that the dust/gas ratio is constant and the grain size distribution is the same everywhere, so that $I \propto \int n_{i} n_{d} d l \propto \int n_{i}^{2}$.

We have studied both the (nearly) full sky and smaller independent regions of interest including the Gum Nebula, the Oph complex, and the Orion Nebula. For all regions, the $\mathrm{H} \alpha$-correlated emission is well fit by a linear combination of an $I_{\nu} \propto \nu^{-0.15}$ power law for free-free and a Draine \& Lazarian (1998) WIM spinning dust model shifted in frequency by a factor of 2 and with only a minimal adjustment in amplitude. Specifically, the spinning dust component is $\sim 4-7$ times weaker than the free-free component.

The null hypothesis that a free-free only spectrum is consistent with the data is ruled out at $\geq 8 \sigma$ confidence for all regions considered here, despite the substantial noise in the data. Furthermore, we have shown that the bump in the $\mathrm{H} \alpha$-correlated emission spectrum is not due to chance cross correlation between the $\mathrm{H} \alpha$ map and the additional foreground templates presented in Dobler \& Finkbeiner (2008) indicating that it is indeed correlated primarily with $\mathrm{H} \alpha$ emission.

Finkbeiner et al. (2002) listed a number of criteria by which the existence of spinning dust could be established. Among them were that the peak frequency must shift as a function of the en- vironment in a way at least qualitatively consistent with DL98. In this work we find in the WMAP data a WIM-correlated component with a peak frequency of $50 \mathrm{GHz}$, about a factor of 2 higher than the peak frequency of the spinning dust component that is spatially correlated with thermal dust emission (i.e., WNM or CNM spinning dust). That this frequency is higher is in accordance with the DL98 models, strongly supporting the spinning dust hypothesis. However, because the shift is larger than expected, a careful assessment of the situation requires that the reason for the size of the shift be determined. If, for example, the WIM dust has smaller grains, then a correlated measurement of $R_{V} \equiv A_{V} / E_{B-V}$ using, e.g., background stars would complete the case for spinning dust.

In summary, there is clearly an additional foreground component correlated with $\mathrm{H} \alpha$ emission and its spectral shape is consistent with spinning dust, although at a somewhat higher frequency than expected. However, the increased sensitivity, frequency coverage, and finer spatial resolution of future missions such as Planck may provide the necessary evidence.

We acknowledge helpful discussions with Bruce Draine, Gary Hinshaw, and Joanna Dunkley. Some of the results in this paper were derived using HEALPix ${ }^{7}$ (Górski et al. 2005). This research made use of the NASA Astrophysics Data System (ADS) and the IDL Astronomy User's Library at Goddard. ${ }^{8}$ D. P. F. and G. D. are supported in part by NASA LTSA grant NAG5-12972.

\footnotetext{
7 The HEALPix Web page is http://healpix.jpl.nasa.gov.

8 Available at http://idlastro.gsfc.nasa.gov.
}

Bennett, C. L., et al. 2003, ApJS, 148, 97

Boughn, S. P., \& Pober, J. C. 2007, ApJ, 661, 938

de Oliveira-Costa, A., Kogut, A., Devlin, M. J., Netterfield, C. B., Page, L. A., \& Wollack, E. J. 1997, ApJ, 482, L17

de Oliveira-Costa, A., Tegmark, M., Page, L., \& Boughn, S. 1998, ApJ, 509, L9

de Oliveira-Costa, A., et al. 1999, ApJ, 527, L9

. 2000, ApJ, 542, L5

2002, ApJ, 567, 363

. 2004, ApJ, 606, L89

Dennison, B., Simonetti, J. H., \& Topasna, G. 1998, Publ. Astron. Soc. Australia, 15,147

Dickinson, C., Casassus, S., Pineda, J. L., Pearson, T. J., Readhead, A. C. S., \& Davies, R. D. 2006, ApJ, 643, L111

Dickinson, C., Davies, R. D., \& Davis, R. J. 2003, MNRAS, 341, 369

Dobler, G., \& Finkbeiner, D. P. 2008, ApJ, 680, 1222 (DF08)

Draine, B. T., \& Lazarian, A. 1998, ApJ, 508, 157 1999, ApJ, 512, 740

Erickson, W. C. 1957, ApJ, 126, 480

Ferrara, A., \& Dettmar, R.-J. 1994, ApJ, 427, 155

Finkbeiner, D. P. 2003, ApJS, 146, 407 2004, ApJ, 614, 186

\section{REFERENCES}

Finkbeiner, D. P., Davis, M., \& Schlegel, D. J. 1999, ApJ, 524, 867

Finkbeiner, D. P., Langston, G. I., \& Minter, A. H. 2004, ApJ, 617, 350

Finkbeiner, D. P., Schlegel, D. J., Frank, C., \& Heiles, C. 2002, ApJ, 566, 898

Gaustad, J. E., McCullough, P. R., Rosing, W., \& Van Buren, D. 2001, PASP, 113,1326

Górski, K. M., et al. 2005, ApJ, 622, 759

Haffner, L. M., Reynolds, R. J., Tufte, S. L., Madsen, G. J., Jaehnig, K. P., \& Percival, J. W. 2003, ApJS, 149, 405

Haslam, C. G. T., Stoffel, H., Salter, C. J., \& Wilson, W. E. 1982, A\&AS, 47, 1 Hinshaw, G., et al. 2007, ApJS, 170, 288

Kogut, A., et al. 1996, ApJ, 464, L5

Langston, G., Minter, A., D’Addario, L., Eberhardt, K., Koski, K., \& Zuber, J. 2000, AJ, 119, 2801

Leitch, E. M., Readhead, A. C. S., Pearson, T. J., \& Myers, S. T. 1997, ApJ, 486, L23

Page, L., et al. 2007, ApJS, 170, 335

Scaife, A. M. M., et al. 2008, MNRAS, 385, 809

Schlegel, D. J., Finkbeiner, D. P., \& Davis, M. 1998, ApJ, 500, 525

Spitzer, L. 1978, Physical Processes in the Interstellar Medium (New York: Wiley) 